\title{
Distributed cognition and distributed language: analysis of cognitive events in the social practice of emergency medicine
}

\author{
SARAH BRO TRASMUNDI
}

This article discusses and evaluates distributed cognition and distributed language, respectively. It argues that a broader understanding of the object of analysis - human interaction and cognition - is needed if the ecology of human interaction is to be embraced in its entirety.

Having discussed the theoretical framework, the article presents analyses of how cognitive events are managed in an emergency ward at a Danish hospital. It uses Cognitive Event Analysis (Steffensen 2013), which focuses on how a system is enabled to achieve cognitive results or outputs, for instance how a medical team reaches a diagnosis, taking into account the rapid timescales of inter-bodily dynamics. The analyses draw on real-life video recordings and investigate how healthcare practitioners manage diagnostic activities in a way that yields cognitive results. It shows how whole-bodied (mis)coordination between an agent and his environment contributes to (dys)functional outcomes. Finally, the article discusses theoretical, methodological and practical implications of a distributed perspective on language and cognition. It concludes that in order to access a cross-section of the human ecology, language activities and cognitive processes must be viewed as distributed and intertwined as well as realised through co-action and coordination in a distributed cognitive system. 